\title{
Participation of customers in Active Demand Side Participation programs under different pricing schemes
}

\author{
Han Xu, Ivana Kockar \\ Department of Electrical and Electronic Engineering \\ University of Strathclyde \\ Glasgow, UK \\ \{han.xu; ivana.kockar\}@strath.ac.uk
}

\begin{abstract}
Operation of the power grid is currently undergoing fundamental and significant changes due to implementation of new technologies and attempts to reduce impact on environment, as well as improve security of supply by diversifying generation mix. This paper outlines a price incentive scheduling tool for consumer-based active demand side management that can prove flexibility necessary for operation of systems with high levels of renewable generation penetration. The scheduling tool helps consumers rescheduling the home appliances corresponding to different pricing notifications based on their energy consumption preferences. The analysis of individual household behaviours is carried out for two price scenarios: (i) predefined electricity price tariff and (ii) forecast real time pricing (RTP) developed on the basis of time series analysis.
\end{abstract}

Index Terms-- active demand side participation; demand side management; household appliance scheduling; energy storage; electricity pricing

\section{NOMENCLATURE}

\section{A. General sets}

$t$

B. Input data

$P_{\min }(t), P_{\max }(t)$

$u_{\text {i.ini }}(t=0)$

$t_{\text {ON.ini }}$

$P_{i}$

$T_{\text {i. op }}, T_{i . \text { off }}^{\max }$

$U_{i \text { ON }}^{\min }, U_{i \text { ON }}^{\max }$

$P_{\text {s.char }}^{\min }(t), P_{\text {s.char }}^{\max }(t)$

$P_{\text {s.dischar }}^{\text {min }}(t), P_{\text {s.dischar }}^{\max }(t)$

$\operatorname{SOC}(t)^{\min }, \operatorname{SOC}(t)^{\max }$

$\pi(t)$

C. Variables

$u_{i}(t)$

$u_{\text {s.char }}(t), u_{\text {s.dischar }}(t)$

$U_{i . O N}$

The development of this research is supported by UK Engineering and Physical Sciences Research Council (EPSRC) through the grant project Autonomous Power System (APS) programme. [EP/I031650/1]

$P_{\text {s.char }}(t), P_{\text {s.dischar }}(t)$
$T_{\text {i. ON }}, T_{\text {i. OFF }}$
$P(t)$
$\operatorname{SOC}(t)$

Storage charging and discharging power at time $t$

Operating/OFF duration of home appliance $i$ since it has been turned on/off, respectively

Total energy consumptions at time $t$

Storage $s$ State of Charge (SOC) at time $t$

\section{INTRODUCTION}

Operation of the power grid is currently undergoing fundamental and significant changes due to implementation of new technologies and attempts to reduce impact on environment, as well as improve security of supply by diversifying generation mix. This includes penetration of renewable generation both at the transmission and distribution levels, together with integration of a demand side flexibility, energy storage and electric vehicles. These changes are also typically associated with a shift towards more flexible decentralised operation of power systems.

A particular emphasis on the operational flexibility in the power network is coming from the demand side. Demand Side Management (DSM) covers a broad range of techniques, which typically refer to the methods applied to manage the consumer side by distribution and transmission network operators. The aim of DSM is to manage the load consumption through peak reduction and load shifting according to generation availability and system constraints [1]. An overview of the benefits and potential applications of DSM at different voltage levels is given in [2] and it includes management of intermittent renewable sources as well as improvement of the network efficiency. In addition, comparisons and analysis of various DSM techniques attracted significant attention by power system community [2]-[5]. Typically, initial DSM techniques were based on a Direct Load Control (DLC) [6]-[8], where demand e.g. home appliances, are reacting to direct remote control signals sent by network/system operators or on changes in system wide parameters such as a system frequency. Thus, under DLC scheme home appliances are scheduled and controlled by an external entity and typically with a relatively short notice. Under current pilot schemes, DLC is typically carried out on specially equipped frequency-responsive load devices, e.g. air conditioners and refrigerators. Implementation of DLC, however, may necessitate evaluation of consumers' tolerance levels towards these direct external interventions, especially if various groups of home appliances are affected.

In order to overcome perceived intrusiveness of DLC approaches which may discourage some customers to take part 
in such programs, DSM methods based on Active Demand Participation (ADP) where customers receive external signals to which they may or may not respond have started to emerge. For example, pricing incentive programs such as the economy 7 tariff in the UK is based on the time of use (ToU) pricing [9], which is usually combined with storage heaters. Similarly, real-time pricing (RTP) is being analysed with demand side in [10]-[11]. Both ToU pricing and dynamic pricing vary electricity rates during different times of a day. However, the calculation and variability of the rates in these two pricing programs are different. ToU pricing typically has predetermined fixed prices for different times of the day. It is based on the production and utility cost without considering the electricity market clearing prices. In contrast, real-time pricing varies throughout the day based on the outcomes of the dayahead or real-time market clearing.

This paper outlines an approach that enables customers to take part in Active Demand Participation program and help them decide how to schedule various appliances within the household. In contrast to the external DLC, the consumerbased Active Demand Participation approach schedules endusers' devices on the basis of customers' preferences and predefined daily electricity consumption patterns. This means that consumers are taking control of their home appliances and all of the decisions are made so to achieve objectives of customers, rather than directly help optimize network operation. To achieve optimized operation, system operator and/or aggregators need to develop control algorithms that will entice customers to respond to external signals, however it is outside of the scope of this paper.

While such approach can lead to suboptimal operation from the point of view of the system and network operators, it is important to acknowledge that they may significantly improve customer engagement and consequently increase energy savings of residential households if customers seek to improve their energy efficiency and reduce energy waste. Typically, price incentive DSM methods assume availability of smart meters which are regarded as enabling technology. They are used for the bi-directional communication between suppliers and customers, to bring necessary signals and information to customers and also be used to monitor consumption and respond to signals. An agent-based model which investigates these interactions and assumes decentralised DSM via smart meters is presented in [12]. It uses day-ahead prices in an attempt to flatten the demand. An energy management system is proposed in [13] with smart meters and smart domestic appliances. An intelligent home energy management system is illustrated in [14], smart meter receives external signals on the amount of energy needs to be curtailed and the curtailment duration. The energy management system will make the decision based on load priorities, temperatures, etc.

Results of some price based DSM pilot programs are given in [15], and include both the DLC and consumer-based ADP approaches. These projects showed an average of $8 \%-15 \%$ peak reductions, which was promising. The results also indicated the potential of residential loads being regarded as a group that should be further investigated to provide demand flexibility.

As a bottom-up approach, ADP relies on customers to make their own decisions regarding when and how much energy to consume. However, it may not be an easy task for an average household, and thus development of tools that will help their engagement is one of the important areas to consider. The aim of this paper is to present a scheduling tool that enables participating customers to automatically respond to the inflow price signals that may vary during a day. The tool seeks to minimize cost of energy while ensuring that preferences stemming from their life-style choices and a need to supply at least certain pre-specified amount of energy are respected. Therefore, it decides on when household appliances will be scheduled. The paper compares outcomes when pre-set electricity tariffs and stochastic dynamic pricing are used.

The paper is organised as follows: Section III outlines the proposed ADP scheduling tool together with the mathematical formulation of the optimization problem. The tool is illustrated on two case studies using two different pricing approaches mentioned above, with results of these analysis presented in Sections IV. This section also briefly outlines the procedure used to forecast stochastic electricity prices by time series analysis.

\section{SCHEDUling TOOL FOR CONSUMER-BASED DSM}

As mentioned above, the main objective of the proposed tool is to enable automatic scheduling of household appliances and to minimize energy payments while maintain condition imposed by the household occupants. It assumes that the customers are participating in an ADP program where price signals are sent by suppliers with expectations that the customer will modify their consumption based on these prices.

Implementation of the scheduling tool in individual households also assumes integration via the smart meter, as illustrated in Fig. 1. This tool is the main component of the 'intelligence box' attached to the smart meter and installed into each individual household.

Inflow price signals are sent by suppliers or aggregators which seek to influence demand at particular time period. These target load levels reflect the objectives they what to achieve. For example, if the aggregator seeks to provide a reserve to the system operator, higher price signals will be sent to reduce demand. On the other hand, if connected wind generators are facing curtailment due to network constraints, the aggregator may seek to increase consumption of some customers. Thus, reduced price will be sent to these customers so that they take advantage of locally available constrained resource, but also allow those generators to produce and reduce curtailment. In the ADP approach consumers in the household can choose whether they are willing to respond to sent price signals and if so when and how. If they are inclined to actively participate into the DSM scheme, their load devices will be rescheduled based on their own requirements. The aim of the tool is to help consumers reduce their energy payments, and fulfil their energy consumption needs at the same time.

The challenge of such a method is to meet consumers' various needs during different time periods, as well as to ensure that the total energy requirement for a specified period of time is met. The consumption preferences should be

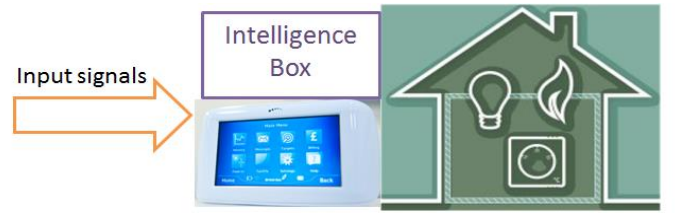

Figure 1. Illustration of DSM program application. 
allocated to each home appliance. The decomposition results for home appliances for the UK demand curve are given in [16]. It also includes the total energy usage amount of each home device during different time slots in a day. Furthermore, the flexibility level of each load device (e.g. electric water heater) also reflects consumers' energy usage patterns. This means that the scheduling tool will contain information on the contribution of each load group to the rescheduling through investigating the load flexibility level.

The flexibility level is divided into three catalogues - high, medium, and low level. Note that here flexibility is regarded as the availability to be rescheduled. Moreover, the operation constraints of the controlled loads and scheduling impact on consumers' life are the criterion for the flexibility level as well. These constraints are controlled via input parameters that are specified by the consumer, and can be chanted to reflect different preferences. For high flexibility loads, consumers are usually unaware when they are operating and modifying consumption according to price signals. These include electric water heater and space heating as well as energy storage. The survey in Fig. 2 [17] shows responses of customers to the questioner on the activities they are engaged during evening and activities they are willing to shift after 9pm. It shows that dishwashers and washing machines have $37.5 \%$ and $65.2 \%$ respondents willing to shift their activities, respectively. These types of appliances are categorized into the medium flexibility group, which will respond to price signals but are less flexible. Finally, the low flexibility appliances group will only respond to DSM program if it does not cause inconvenience to consumers' lives, as the impact of shifting such load is immediately felt. For example, only $0.8 \%$ of customers indicated willingness to shift their cooking activities after 9 p.m., and over $90 \%$ watch TV during 7 p.m. to 9 p.m. in the survey. Therefore, cooking devices and TVs are regard as low flexibility loads. Zero flexibility load appliances are not engaged into DSM program at all, and therefore are not included in the presented model.

\section{A. Problem Formulations}

The objective of the scheduling tool is to minimize customer payments over given period of time. Thus, objective function and constraints are defined as:

$$
\text { Minimize COST }=\sum_{t=1}^{T}\left\{P(t)^{*} \pi(t)\right\}
$$

Subject to

$$
\begin{aligned}
& P(t)=\left\{\sum_{i=1}^{I}\left\{u_{i}(t) * P_{i}\right\}\right\}+u_{\text {s.char }}(t) * P_{\text {s.char }}(t) \\
& -u_{s . d i s c h a r}(t)^{*} P_{s . d i s c h a r}(t) \quad \forall i, \forall t, \forall s \\
& P_{\text {min }}(t) \leq P(t) \leq P_{\text {max }}(t) \quad \forall t
\end{aligned}
$$

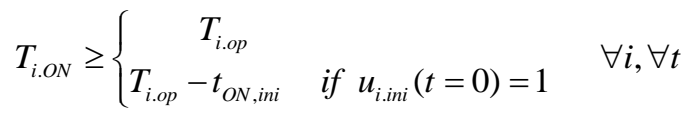

$$
\begin{aligned}
& T_{i . \text { OFF }} \geq\left\{\begin{array}{c}
T_{i . \text { off }}^{\max } \\
T_{i . \text { off }}^{\max }+t_{\text {ON,ini }}
\end{array} \text { if } u_{i . \text { ini }}(t=0)=0 \quad \forall i, \forall t\right. \\
& U_{i . O N}=1^{T} * u_{i}(t) \quad \forall i, \forall t
\end{aligned}
$$

$$
\begin{aligned}
& U_{i . O N}^{\min } \leq U_{i . O N} \leq U_{i . O N}^{\max } \quad \forall i \\
& \operatorname{SOC}(t)=\operatorname{SOC}(t-1)+u_{\text {s.char }}(t) * P_{\text {s.char }}(t) \\
& -u_{\text {s.dischar }}(t) * P_{\text {s.dischar }}(t) \quad \forall t, \forall s \\
& \operatorname{SOC}(t)^{\min } \leq S O C(t) \leq S O C(t)^{\max } \quad \forall t \\
& u_{s . c h a r}(t) * P_{s . c h a r}^{\min }(t) \leq P_{\text {s.char }}(t) \quad \forall t, \forall s \\
& u_{\text {s.char }}(t) * P_{s . c h a r}^{\max }(t) \geq P_{\text {s.char }}(t) \quad \forall t, \forall s \\
& u_{\text {s.dischar }}(t) * P_{\text {s.dischar }}^{\min }(t) \leq P_{\text {s.dischar }}(t) \quad \forall t, \forall s \\
& u_{\text {s.dischar }}(t) * P_{\text {s.dischar }}^{\max }(t) \geq P_{\text {s.dischar }}(t) \quad \forall t, \forall s \\
& u_{i}(t) \in[0,1] \quad \forall i, \forall t \\
& u_{i . i n i}(t=0)=\left\{\begin{array}{ll}
0 & t_{O N, i n i} \leq 0 \\
1 & t_{O N, i n i}>0
\end{array} \quad \forall i, \forall t\right. \\
& u_{\text {s.char }}(t) \in[0,1] \quad \forall t, \forall s \\
& u_{\text {s.dischar }}(t) \in[0,1] \quad \forall t, \forall s \\
& u_{\text {s.char }}(t)+u_{\text {s.dischar }}(t)=1 \quad \forall t, \forall s
\end{aligned}
$$

In the above constraints, power consumption in the household in (2) considers the charging and discharging activities of energy storage device. Constraint (3) defines the overall power consumption limitations, which is used in some countries to limit the amount of power that a household can draw at any instant in time. Some appliances have the minimum operating time and maximum switching off period. The operating duration and off time of each appliance is determined by the time period it has been turned on and off, respectively. These are defined in (4) and (5), and they also respects initial status of home devices provided as an input. For instance, washing machine has few cycles which, once started, should not be interrupted. The total running time duration of the devices is calculated by (6), with its upper and lower limitations enforced by (7). The relationship between the state of charge (SOC) of the storage, levels of energy charging and discharging, as well as previous SOC state is given in (8). Since every type of energy storage has its own maximum level of energy stored, the limitations of the maximum and minimum SOC are defined in (9), while the limitations on the levels of charging and discharging energy per hour are expressed in (10)-(13). Binary variables in (14) are used to decide on/off

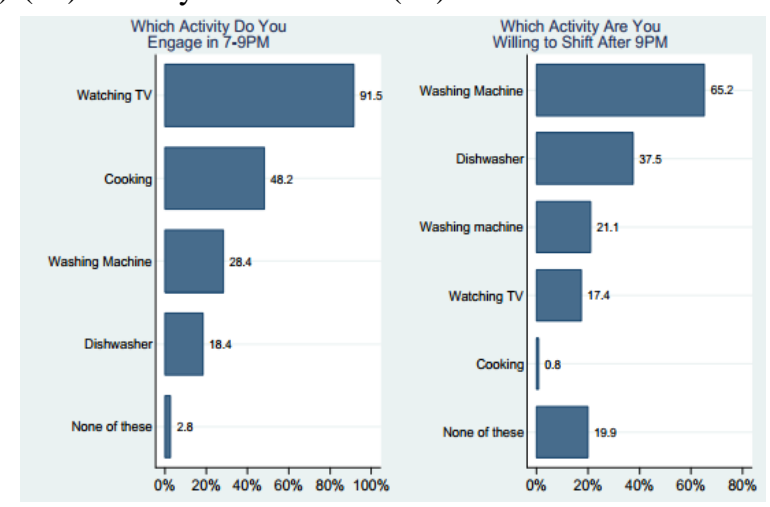

Figure 2. EPRG survey on potential load shifting. [17] 
status for each home device that included in the scheduling tool. The initial status of each load is presented by the binary number in (15). Additional binary variables defined in (16) and (17) are used for implying the charging and discharging status of the energy storage device. Thus the charging/discharging status of storage is determined by the scheduling tool, with the control that only one of these activities can be active as defined by (18). Note that parameters in all of these constraints are decided by consumers and are set as input into the scheduling tool. For example, the cooking devices (exclude toasters) are assumed to operate only during the evening time, and will be used for a relatively short period of time.

\section{ANALYSIS OF TWO DIFFERENT PRICING SCHEMES}

In the following test cases, eight typical home appliances at various flexibility levels are employed: electric water heater, tumble dryer, washing machine, cooking devices, dishwasher, refrigerator, space heater and TV. This is the base case household (case I), which will be compared to the same household but with an addition of the battery storage (case II). The cases are solved using Fico Xpress solver [18], which is used for mixed-integer programming problems, and applies branch and bound method to obtain optimal solutions. Results of testing two different price incentive signals for 24 hours are presented and discussed next. These include pre-set price tariffs and stochastic prices. For example, space heater should operate between 6 hours and 10 hours during a day in the instance of October. Washing machine runs maximum 1 hour every day and the washing process cannot be interrupted.

\section{A. Pre-set Electricity Price Schemes}

To date, pre-set/fixed electricity price tariffs are commonly applied for pricing energy for residential houses. The left side of Fig. 3 illustrates the electricity price curve for the pre-set price scheme tested, which is designed on the basis of economy 7 electricity tariffs in the UK. The first seven hours from midnight are configured as half price of the normal price during the rest of the day. Right side of the Fig. 3 gives result from the scheduling tool for the base case I (upper) and case II with the addition of batteries (lower graph). The common result for both cases is that the flexible home appliances with larger energy consumptions are scheduled towards the lower electricity price time periods. This includes charging of battery storage in case II. The charging and discharging activities of battery storage are shown in the lower right hand figure in Fig. 3. The initial SOC of the storage is assumed to be $1.8 \mathrm{kw}$, which is the original energy power stored in the storage before any activity of charging or discharging started. By comparing the scheduling results for two systems, the load curve of the

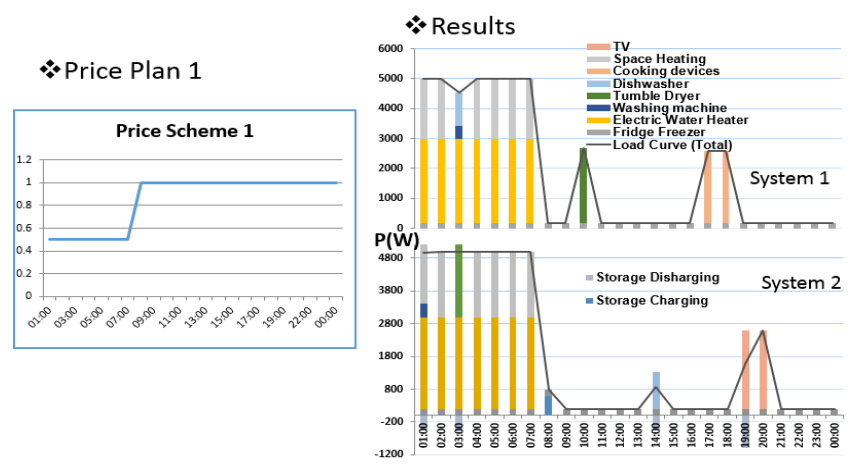

Figure 3. Economy 7 tariff and rescheduled loads case II has smaller peaks since it makes the most of the off peak electricity prices through combination with energy storage. As a result, the second system with battery storage has more energy savings.

\section{B. Stochastic Electricity Price Scenarios}

Fixed pricing will not be the most appropriate for the purpose increasing renewable energy integration. Rather, a dynamic pricing approach where prices calculated at frequent time intervals so to reflect levels of available inexpensive renewable generation as well as network conditions seems more suitable, although it is more complex to implement. The second type of price incentive signal analysed here is the stochastic dynamic pricing. Electricity prices are timedependent and are affected by certain stochastic factors. Different price forecasting techniques are compared and illustrated in [19]. ARIMA process is the technique used in this paper for forecasting stochastic pricing based on day-ahead electricity rates. ARIMA approach is chosen because of its independence upon the network conditions, e.g. the variation on demand consumptions, the changing renewable generation capacity, etc. Furthermore, Box-Jenkins modelling approach [20] is used for establishing the ARIMA time series model, with the electricity price data based on PJM [21]. The parameters of both auto regressive and moving average part are adjusted according to PJM day-ahead RTP data. The root mean square error (RMSE) and the mean absolute error (MAE) is the criterion indicating good models [22]. $\operatorname{ARMA}(4,0,4)$ is the final result acquired for forecasting future RTP. The RMSE value of the model is around 0.2, and the MAE is 0.15. All the values above indicate a good ARMA model is developed.

There are various approaches for generating forecasting scenarios. Typically, scenarios are generated by probabilitybased methods, e.g. Monte Carlo sampling. In this paper, forecasting scenarios of electricity prices in the next 24 hours are created on the basis of ARMA time series model. They are generated through randomly sampling the distribution of one part in price rates that is not easily decipherable [23], which follows a Gaussian distribution with zero mean value and constant deviation. All the forecasted scenarios could be the future trend of stochastic price for a day due to the increasing penetration of renewable resources and DERs. A representative forecast scenario is presented in Fig.4 as the upper curve. The dynamic pricing scenarios are tested into individual residential household, which means the price term is altered to the forecast scenarios in (1). The scheduled load curve result corresponding to the RTP scenario is shown in Fig. 4 as the bottom curve.

Only the case II with battery storage device is employed for observing virtual consumers' responses. When there is a relatively large error between the forecast prices and real rates

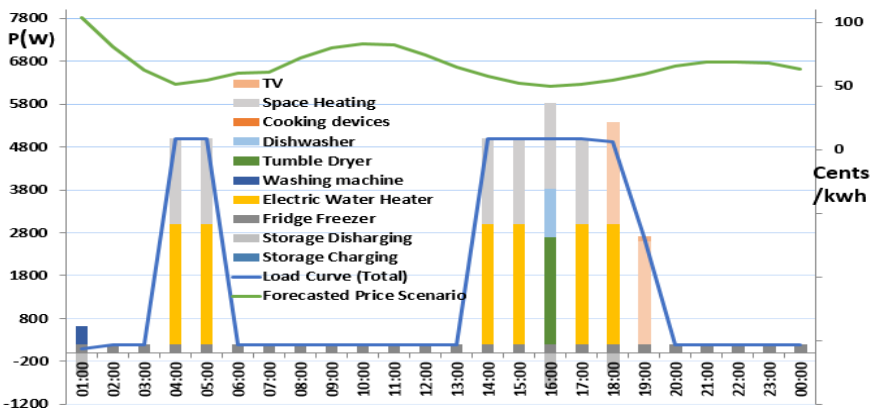

Figure 4. Forecasting price scenario 1 and rescheduled loads 


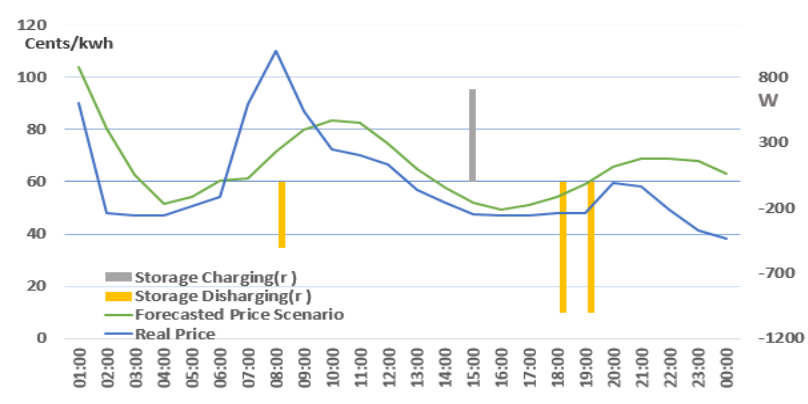

Figure 5. Energy storage activity - compensate forecast error

in Fig. 5, the storage device can be used for compensating the errors. This is achieved by using rolling planning technique to enable the scheduling tool to respond to real-time data. However, the application of rolling planning is out of the scope of this paper. The forecast scenario shares an overall similar trend with real energy price, except a relatively large error between two price sets during the morning peak time. The storage device discharges when the big difference happens to compensate the forecasting errors. After that, the energy storage chooses low price time to charge and discharges during the evening peak duration. Due to the storage is not limited to store a certain level of energy at the end of the day, it consumes all of its stored electricity in this case. Therefore, battery storage contributes to an increased level of flexibility when consumers are facing diverse RTP values.

\section{CONCLUSION AND FUTURE WORK}

The paper proposes a price incentive scheduling tool for consumer-based active DSM that can prove flexibility necessary for operation of systems with high levels of renewable generation penetration. We are assuming demand side actively participations to drive the practical application into individual residential household. The tool helps consumers rescheduling the home appliances corresponding to different pricing notifications based on their energy consumption preferences, including the predefined electricity price tariff and forecast RTP scenarios developed on the basis of time series analysis. And energy storage device is included in the test household model to provide certain flexibility level particular for the case of stochastic electricity prices. Both two case studies have sufficient evidence showing the consumer-based tool succeeded in achieve its goal. Therefore, the tool makes it possible for consumers to monitor the price signals intelligently and achieve energy bill savings automatically.

Potential enhancements of this consumer-cased scheduling tool could be made in two aspects, i) add stochastic characteristic into the scheduling tool to achieve dynamic control of home appliances when facing uncertain real-time network conditions; ii) implement the scheduling tool to respond to household environmental conditions in a physicallybased modelling framework.

\section{REFERENCES}

[1] N. Zhang, L. F. Ochoa, D. S. Kirschen, "Investigating the impact of demand side management on residential customers," in Innovative Smart Grid Technologies (ISGT Europe), 2011 2nd IEEE PES International Conference and Exhibition on, pp.1-6.

[2] G. Strbac, "Demand side management: benefits and challenges," Energy policy, vol. 36, pp.4419-4426,Dec. 2008.
[3] M.H.Albadi,E.El-Saadany,"A summary of demand response in electricity markets,"Electric Power Systems Research,vol. 78,pp. 19891996, Nov. 2008.

[4] Y. Tan, D. Kirschen (2007). "Classification of control for demand-side participation," University of Manchester. [Online]. Available: http://www.ee.washington.edu/research/real/Library/Reports/Classificati on_of_Demand-Side_Controls.pdf

[5] Q. Zhang, J. Li (2012). "Demand response in electricity markets: a review," In European Energy Market (EEM), 2012 9th International Conference on the, pp. 1-8.

[6] K. -H. Ng, G. B. Sheble, "Direct load control-a profit-based load management using linear programming," IEEE Trans. Power Systems, vol.13, pp. 688-694, May 1998.

[7] N. Ruiz, I. Cobelo, J. Oyarzabal, "A direct load control model for virtual power plant management," IEEE Trans. Power Systems, vol. 24, pp. 959-966, May 2009.

[8] R.Teive,S.Vilvert, "Demand side management for residential consumers by using direct control on the loads," in 2002 5th International Conf. on Power System Management and Control,pp.233-237.

[9] J. Torriti, "Price-based demand side management: Assessing the impacts of time-of-use tariffs on residential electricity demand and peak shifting in Northern Italy," Energy, vol. 44, pp. 576-583, Aug. 2012.

[10] Y. Ding, S. Pineda, P. Nyeng, J. Ostergaard, E. M. Larsen, Q Wu, "Real-time market concept architecture for EcoGrid EU-A prototype for European smart grids," IEEE Transactions on Smart Grid, vol. 4(4), pp. 2006-2016, Dec 2013.

[11] A.-H.Mohsenian-Rad, A. Leon-Garcia, "Optimal residential load control with price prediction in real-time electricity pricing environments," IEEE Transactions on Smart Grid, vol. 1, pp. 120-133, Sept. 2010.

[12] S. D. Ramchurn, P. Vytelingum, A. Rogers, N. Jennings, "Agent-based control for decentralised demand side management in the smart grid," In The 10th International Conference on Autonomous Agents and Multiagent Systems-Volume 1, pp. 5-12. International Foundation for Autonomous Agents and Multiagent Systems, 2011.

[13] W. T. Li, C. Yuen, N. U. Hassan, W. Tushar, C. K. Wen, K. L.Wood, et al, "Demand Response Management for Residential Smart Grid: From Theory to Practice," IEEE Access, vol.3, pp.2431-2440, Dec 2015.

[14] M. Pipattanasomporn, M. Kuzlu, S. Rahman, "An algorithm for intelligent home energy management and demand response analysis," IEEE Transactions on Smart Grid, vol.3, pp. 2166-2173, Dec 2012.

[15] Department of Energy and Climate Change (2012) "Demand side response in the domestic sector- a literature review of major trials (Final Report)",UK. [Online] Available: https://www.gov.uk/government/uplo ads/system/uploads/attachment_data/file/48552/5756-demand-side-resp onse-in-the-domestic-sector-a-lit.pdf]

[16] V. Silva, V. Stanojevic, M. Aunedi, D. Pudjianto, G. Strbac (2011). "7 Smart domestic appliances as enabling technology for demand-side integration: modelling, value and drivers," In The Future of Electricity Demand: Customers, Citizens and Loads, pp. 185-211.

[17] L. Platchkov, M. G. Pollitt, D. Reiner, I. Shaorshadze, "2010 EPRG Public opinion survey: policy preferences and energy saving measures," Cambridge, Electricity Policy Research Group, Faculty of Economics, University of Cambridge.

[18] Fico Xpress [Online] Available: http://www.fico.com/en/products/ficoxpress-optimization-suite/

[19] A.J.Conejo,J.Contreras, R.Espinola, M.A.Plazas, "Forecasting electricity prices for a day-ahead pool-based electric energy market," International Journal of Forecasting,vol.21,pp. 435-462,July-Nov. 2005.

[20] G.E.Box, G.M.Jenkins, G.C.Reinsel, Time series analysis: forecasting and control, John Wiley \& Sons, 2013.

[21] https://www.pjm.com/

[22] R.J.Hyndman,A.B.Koehler,"Another look at measures of forecast accuracy,"International Journal of Forecasting,vol.22,pp.679-688,Oct.Dec. 2006.

[23] A. J. Conejo, M. Carrión, J. M. Morales, Decision making under uncertainty in electricity markets, New York: Springer.2010 